\title{
Compressed Radiological Images and Workstation Viewing
}

\author{
Dennis L. Wilson
}

The viewing of radiological images on workstations has peculiarities that must be taken into account in the design of a compression technique. The images may be manipulated on a workstation to change the contrast, to change the center of the brightness levels that are viewed, and even to invert the images. Because of the possible consequences of losing information in a medical application, bit-preserving compression is used for the images used for diagnosis. However, for archiving, the images may be compressed to $10 \%$ of their original size. A compression technique based on the discrete cosine transform takes the viewing factors into account by compressing the changes in the local brightness levels. The compression technique is a variation of the Consultive Committee on International Telephony and Telegraphy Joint Photograph Experts Group compression that suppresses the blocking of the discrete cosine transform except in areas of very high contrast.

KEY WORDS: storage and retrieval, compression, discrete cosine transform, Huffman coding.

$\mathbf{R}^{A}$ ADIOLOGICAL IMAGES on workstations must preserve all original information to be accepted by the medical community. The typical radiological image is different from other images in several ways:

1. The images are very low-contrast images locally. Even the transition over the edge of a bone does not change the image intensity by a large amount. The dynamic range over the whole image, however, may be quite large.

2. Much of the information is in the local variation of the image intensity. The overall picture provides a context within which the information is imbedded, but the pathology is visible as local variations.

3. The images are subject to large changes in the individual pixel intensity as the radiologist views the image at a workstation. The radiologist may change the contrast and the center of the contrast range to see the

From the Loral Western Development Labs, San Jose, CA. Address reprints to Dennis L. Wilson, PhD, Loral Western Laboratories, 3200 Zanker Rd, San Jose, CA 95134.

Reprinted with permission from Medical Imaging VI: Picture Archiving and Communications Systems, Society of PhotoOptical Instrumentation Engineers, 1992.

0897-1889/92/0503-0004\$03.00/0 details of the image. In the extreme, the radiologist may invert the image by changing white to black.

4. The images are very large, typically more than $1,000 \times 1,200$ pixels $(1 \mathrm{~K} \times 1.2 \mathrm{~K})$ and up to $2 \mathrm{~K} \times 2.5 \mathrm{~K}$.

5. The image dynamic range may be as large as 12 bits.

6. The image will be viewed at a number of magnifications from 1:4 minified to $8: 1$ zoomed.

A number of techniques are available for compression of images ranging from wavelet coding to transform coding. ${ }^{1} \mathrm{~A}$ transform coding technique that has been used for a number of years is discrete cosine transform (DCT) coding, which has been incorporated into the widely accepted coding standard of the Consultive Committee on International Telephony and Telegraphy Joint Photography Experts Group (CCITT JPEG). ${ }^{2}$

The CCITT JPEG standard uses an $8 \times 8$ DCT in the image compression. There are 8 bits in the image. The blocking effect of the DCT is unacceptable as an artifact over the entire image. If a typical CCITT JPEG compressed image is examined in detail, the blocking of the $8 \times 8$ DCT will be visible over the entire image. The details of the image are visible through the blocking, but the distraction of the blocking over the entire image is unacceptable. Often a radiologist is looking at an image that has been zoomed and in which the contrast has been arranged; the blocks would interfere with the view of the image.

In addition, the dynamic range of 12 bits cannot be squeezed into the 8 bits typically available on a workstation's screen. If the radiological images were viewed in the same way that a television image is viewed, the dynamic range could be compressed to 8 bits by a simple nonlinear mapping of the image intensities. A typical mapping is logarithmic. With this scheme, the image intensities on the display are proportional to the logarithm of the original image intensities. Because the image contrast and intensity center can be varied, including invert- 
ing the pixel values, such a nonlinear mapping is not appropriate. No companding of the image intensity values is tolerable, because the compression from 12 bits will introduce large artifacts at some level of contrast or center for the displayed intensity values. The workstation screen dynamic range of 8 bits matches the characteristic of the human eye. The eye adapts over a wide range of brightness, but at any one general brightness level, the eye does not see more than approximately 256 levels of gray. ${ }^{3}$

Two types of compression used in radiology are lossless compression and lossy compression. The lossless compression returns exactly the same image as the original when the compression is removed, and may be applied to images before they have been viewed by a radiologist. The lossy compression will achieve high compression ratios but will not return exactly the same image as the original. The lossy compressed image is to be used for archiving images for later comparison with a new image. The difference between the original image and the returned compressed image is the compression noise. The compression algorithm is selected to make the compression noise as imperceptible as possible.

The characteristics of the eye have some effect on the perception of the compression noise. The eye can average out uncorrelated high-frequency noise. The radiological image compression proposed will have compression noise pushed to the high frequencies where it will be averaged by the eye.

The compression algorithm is to operate on a radiological image. The image is to be in the American College of Radiology-National Electrical Manufacturers Association ${ }^{4}$ image format. The image is to be rectangular, organized by rows and columns of pixels. The number of rows may be different from the number of columns. Each pixel of the image will be represented by a 16-bit integer image intensity. The dynamic range of the image may be from 8 bits to 12 bits. Bits 0 through 11 are pixel data. Bits 12 through 15 are reserved for annotation.

\section{LOSSLESS COMPRESSION}

Lossless compression is performed using a simple differential pulse code modulation

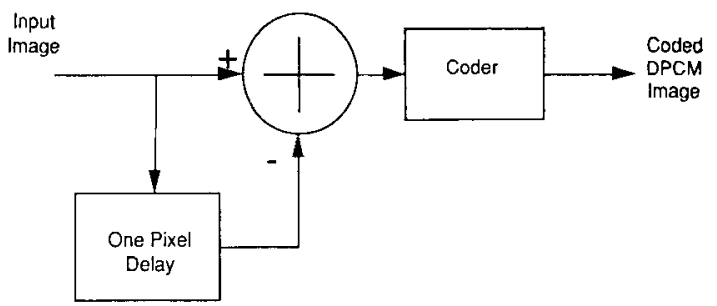

Fig 1. The DPCM coding used for lossless compression is a very simple scheme using only the previous pixel as the predictor of the pixel to be encoded.

(DPCM) scheme with an approximate Huffman coder. A block diagram of the encoder is shown in Fig 1. The difference between adjacent pixels is formed and encoded. The processing wraps at the end of the row to the first pixel of the next row.

An approximate Huffman coding scheme, shown in Table 1, is used. The coded output is a prefix code followed by a pixel value. The prefix code designates the range of the following pixel value, and the value is encoded with a varying number of bits, depending on the range. Values near zero use a small number of bits, whereas large values use the full 12 bits of the dynamic range of the input image.

The expansion of the compressed image is performed as shown in Fig 2. The Huffman code is removed, restoring the DPCM difference values. The pixels are restored by adding successive sample values. The DPCM coder and the DCPM decoder are very simple and are suitable for implementation in a single-gate array or in an application-specific integrated-circuit chip. The compression can be performed very quickly, increasing the effective communication rate and storage capacity by the compression ratio, typi-

Tabje 1. The Huffman Code for the Differential Pulse Code

\begin{tabular}{|c|c|c|c|}
\hline \multicolumn{4}{|c|}{ Modulation Images } \\
\hline Code & Length & $\begin{array}{l}\text { No. of } \\
\text { Codes }\end{array}$ & Value \\
\hline 00 & 2 & 1 & 0 \\
\hline $01 \times x \times x$ & 6 & 16 & $1+x \times x x$ \\
\hline $10 \times x \times x$ & 6 & 16 & $-1-x \times x x$ \\
\hline $1100 \times x \times x x$ & 9 & 32 & $17+x \times x \times x$ \\
\hline $1101 \times x \times x x$ & 9 & 32 & $-17-x \times x \times x$ \\
\hline $11100 \times x \times x \times x$ & 11 & 64 & $49+x \times x \times x x$ \\
\hline$\uparrow 1101 \times x \times x \times x$ & 11 & 64 & $-49-x \times \times \times x \times$ \\
\hline $111101 \times x \times x \times x \times x \times x \times x \times x x$ & 20 & $2^{14}$ & $x X X X X X X X X X X X X X X X$ \\
\hline 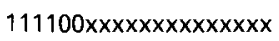 & 20 & $2^{14}$ & $-x x x x x X X X X X X X X X X X x$ \\
\hline
\end{tabular}




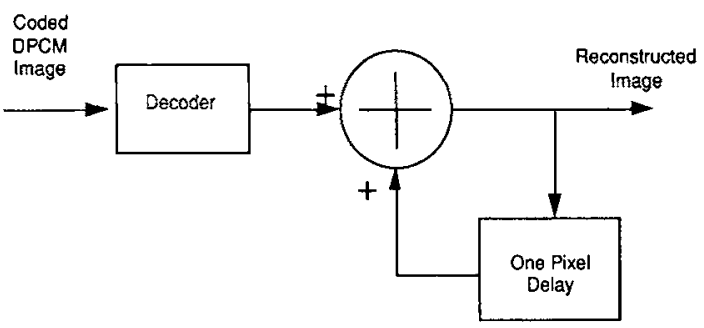

Fig 2. The recovery of the lossless compressed image is equally simple, requiring no multiplies and one add to reconstruct the image.

cally a factor of 2.5 to 3.0 for radiological images.

\section{LOSSY COMPRESSION}

The basic compression scheme is shown in Fig 3. The suppression of the artifacts due to the blocking is accomplished by the use of a difference image. The original image is downsampled and then interpolated back to the original size. The difference between the smoothed image and the original image is then compressed using the $8 \times 8$ DCT with 12-bit data. The major artifacts are a result of the mismatching of the low-frequency components of the image at the edges of the blocks. Except in areas of very large dynamic changes, when the low frequencies are removed from consideration by subtracting the low-frequency components, the artifacts disappear.

The DCT yields a set of transform coefficients that roughly describe the energy levels at frequencies in the row and column directions. The eye is less sensitive to noise at the high frequency, permitting coarser quantization at the higher frequencies, accomplished by frequency weighting the transform coefficients.

\section{THE DOWN-SAMPLED IMAGE}

The first step in the compression is to form the low-pass down-sampled image. The lowpass image may be down-sampled because it has a limited frequency range. The down-sampled image will have 1 pixel for every 4 pixels of the original image in each dimension; therefore, the number of pixels in the down-sampled image will be reduced by a factor of 16 from the number in the original image. The downsampling and subsequent interpolation back to the full-sized image must be performed carefully to form a seamless connection across the blocks. A suitable filter for down-sampling is a Blackman window with a span of 11 pixels, as shown in Fig 4. A Blackman window with a span of 13 pixels is used for the interpolation. For a discussion of the Blackman window, see Parks and Burris. ${ }^{5}$

The down-sampling is performed in two steps, first in the row dimension and then in the column direction. Although a composite filter that performs the complete down-sample operation in one step could be performed, the number of multiply/adds required is greater than in the approach of one dimension at a time.

The interpolation is performed in the row and column directions simultaneously. The interpolation can be viewed as inserting pixels in the down-sampled image by adding pixels with zero value, then performing an interpolating filter

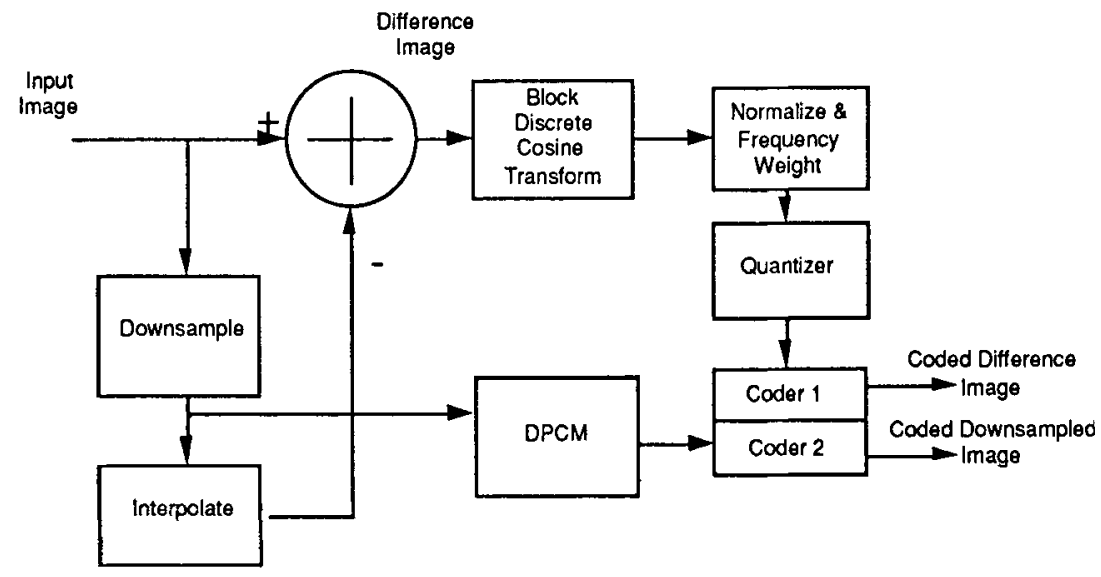

Fig 3. A down-sample-interpolate process is used to eliminate the low-frequency components from the discrete cosine transform operation. 


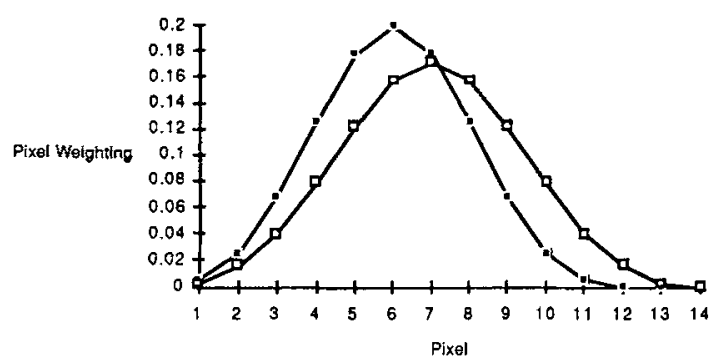

Fig 4. The filtering functions are simple Blackman window functions with no overshoot and consequently no possibility of generating negative numbers. $\square, 11$-point window; $\square$, 13-point window.

operation. The filter will be the Blackman window with a span of 13 . The actual number of multiply/adds required to build the image is limited, because most of the pixel values are zero.

The coding of the down-sampled image is a lossless coding as described above. Successive pixel differences are formed and are coded using the DPCM-approximate Huffman code. The size of the compressed down-sampled image is the size of the original image divided by 16 because of the down-sampling and divided by another factor of 2.5 to 3.0 because of the coding. The resulting storage size for the downsampled image is a factor of 40 to 48 times smaller than the storage required for the original image.

\section{DIFFERENCE IMAGE CODING}

The difference image will be zero-pixel padded to form a full block on the right and bottom of the image. The DCT will be performed on the augmented image to form the DCT coefficients in $8 \times 8$ blocks.

The first step in the compression is to set the quantization level. If the DCT coefficients are coarsely quantized, they will require few bits to encode, whereas if they are finely quantized, they will require many. To make the quantization noise relative to the magnitude of the local variations, the total energy in the transform block is normalized before being weighted, quantized, and coded. The normalization is accomplished by finding the sum of the squares of the cosine transform coefficients to determine the block energy. The gain is determined so that the block energy will be equal to a constant. This gain is applied to each of the coefficients of the transform so that the energy in the block is changed to a constant value called the normalization constant. The normalization constant is limited at each end of the dynamic range; ie, if the normalization constant is larger than a threshold, it is set equal to that threshold. Similarly, if the normalization constant is smaller than a second threshold, it is set equal to that second threshold.

The coefficient normalization step continues by forming the square root of the sum of the squares of the DCT coefficients. A multiplier that will normalize the energy of the block of DCT coefficients is found by dividing the normalized energy constant, a parameter of the compression, by the energy in the block. The normalizing multiplier will be limited both above and below as described above. Each of the coefficient values will be multiplied by the resulting value.

A second step in the compression of the difference image is the elimination of DCT coefficients that have so little energy that they may be ignored: the high-frequency coefficients. The determination of which high-frequency coefficients are to be eliminated is made before the frequency weighting is performed. A zig zag pattern is followed starting from the upper left corner of the block, and coefficients are added until the energy is the selected percentage of the total block energy.

One of the parameters of the compression is the percent of the coefficient energy that is encoded. The coefficients are encoded one at a time in the pattern shown in Fig 5. The encoding is stopped when the amount of energy is greater than or equal to the percent energy threshold. The deletion of coefficients eliminates the highest frequencies where very little energy is expected.

A third step in the compression of the difference image is the frequency weighting of the DCT coefficients. The frequency weighting is performed after the normalization and before the quantization of the coefficients. The frequency weight is a simple exponential weighting function:

$$
\mathrm{W}(\text { row }, \text { column })=\mathrm{e}^{-(\text {row }+ \text { column }) / c)},
$$




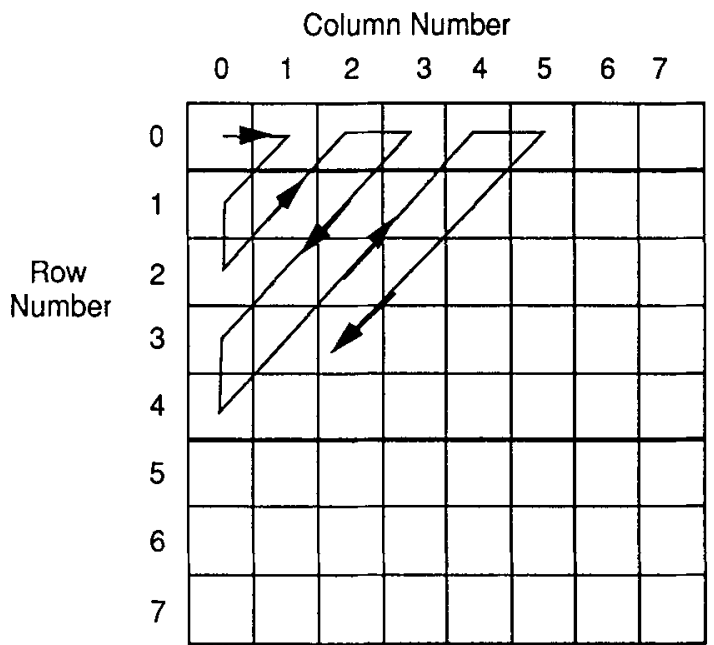

Fig 5. Encoding of coefficients is performed starting at the lowest frequency and proceeding toward the higher frequencies until the desired percent of the energy has been encoded.

where $W$ is the weight, row is the number of the row in the block from 0 to 7 , column is the number of the column within the block from 0 to 7 , and $c$ is the weighting parameter.

After the frequency weighting, the DCT coefficients will be quantized by rounding to form integers. The integers are coded using the DCT-approximate Huffman code.

The Huffman code used for encoding the DCT coefficients is shown in Table 2. The Huffman code uses short code words to transmit coefficient values that happen often and longer code words to transmit coefficient values that are less probable. The average code word length is smaller using this approach than when using a fixed-length code word.

\section{EXPANSION OF THE IMAGE ON RECOVERY FROM THE ARCHIVE}

The expansion of the image follows the inverse of the compression functions. The blocks of the expansion are shown in Fig 6. The

Table 2. Huffman Code for the DCT of the Difference Image

\begin{tabular}{|c|c|c|c|}
\hline Code & Length & $\begin{array}{l}\text { No. of } \\
\text { Codes }\end{array}$ & Value \\
\hline 0 & 1 & 1 & 0 \\
\hline $10 x$ & 3 & 2 & $1,-1(x=0,1)$ \\
\hline $1101 \times x x$ & 7 & 8 & $2+x x x$ \\
\hline $1110 \times x x$ & 7 & 8 & $-2-x x x$ \\
\hline $111101 \times x \times \times x \times \times x \times x \times x \times x$ & 20 & $2^{14}$ & $x x x x x x x x x x x x x x$ \\
\hline $111100 \times x \times \times \times \times \times x \times x \times x \times x$ & 20 & $2^{14}$ & $-x x x \times x x \times x x x x x \times x$ \\
\hline
\end{tabular}

minified image and the normalization value are recovered. The minified image is recovered by performing the bit-preserving reconstruction of the image as shown in Fig 6. The difference image is found by recovering the quantized DCT coefficients using the block energy value recorded in the second word of the compressed block. The inverse DCT is then performed and the frequency weighting is removed, resulting in the difference image. The minified image is then interpolated back to full size and added to the difference image to recover the approximation to the original image.

The DPCM processing of the down-sampled image is shown in more detail in Fig 7. The simplicity of the DPCM encoder is maintained through the DPCM decoder. The result is the recovered down-sampled image that is to be interpolated back to the full size and added to the difference image from the cosine transform processing.

\section{ANNOTATION COMPRESSION}

The images may have attached annotations. These annotation would appear in the top bits of the image word, bits 12 through 15 . Each of the bits will constitute a separate annotation plane. The compression of the annotation will begin by collapsing the four possible planes to one plane. The collapsing function will be a simple "OR" of the four bits of each pixel. The resulting one bit will be compressed using runlength encoding of the single bit-plane image. For many images there will be no annotation. For those images that have annotation, the annotation will be very limited. As a result, there will be large expanses of the annotation image plane that are zero.

The sparse image will be encoded using the compression of CCITT group IV for facsimile. The encoding is a run-length coding scheme with a built-in Huffman code.

\section{COMPRESSED IMAGE FORMAT}

The compressed image has two or three segments, depending on whether the compression is lossless or lossy. There are two segments for the lossless compression, the compressed image and the compressed annotation. The 


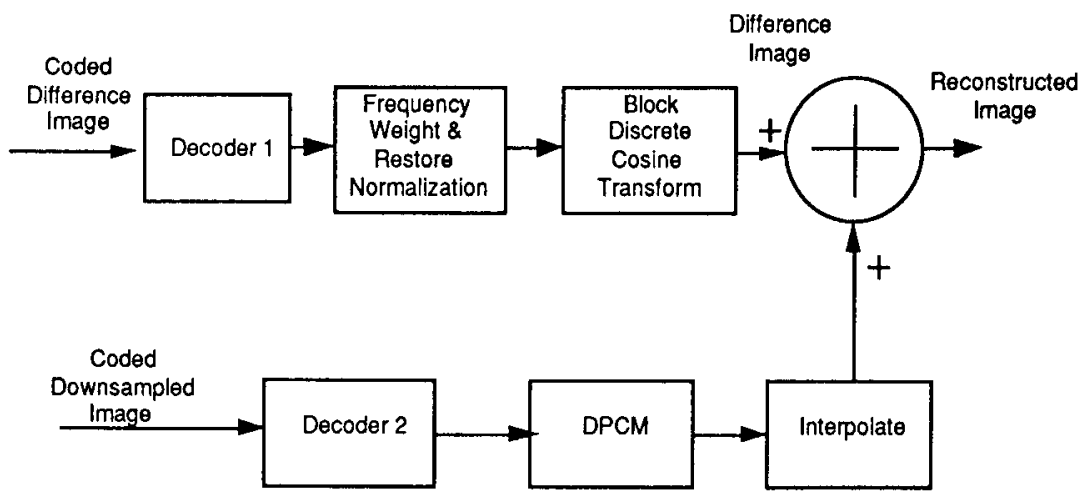

Fig 6. The expansion uses the minified image and the recovered difference image to reconstruct the original image.

compressed image and the compressed annotation are each variable-length strings of bits packed into bytes and zero-padded to make a complete byte at the end.

For the lossy compression there are three segments, the minified image appropriately coded, the DCT-compressed difference image, and the annotation. The first segment, the minified image, is coded the same as it would be for the lossless compression, a variable-length string of bits packed into bytes and zero-padded to a full byte. The compressed difference image will consist of a variable-length string of bits packed into bytes and padded as appropriate. Each block of the large image will consist of a 6-bit word indicating the number of coefficients retained, a 2-bit word indicating the energy level of the block in the difference image, and the remaining Huffman-coded DCT coefficients. The image header will contain the compression parameter described below, the number of rows, and the number of columns of the image.

The down-sampled image is encoded using a simple DPCM scheme as shown in Fig 3. Successive differences in the pixel values are encoded using the DPCM Huffman encoder. The use of

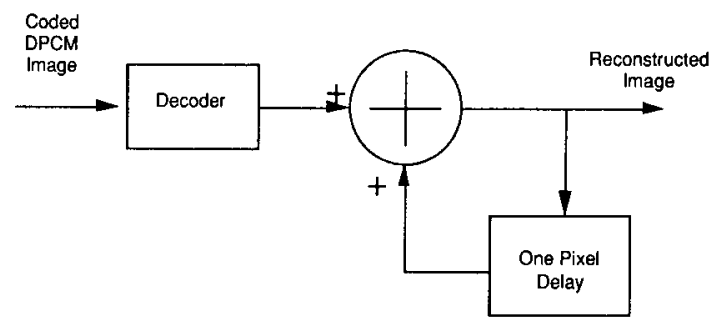

Fig 7. The recovery of the down-sampled image is as simple as the compression and is exactly the same as the recovery for the lossless compression. anything more than the very simple encoding is not warranted. The small image has $1 / 16$ the number of pixels of the large image. The use of the Huffman coder reduces the number of bits by another factor of approximately 2.5 , depending on the image. The result is a compression ratio of 40:1 for the small image. Compression of the difference image by a factor of $40: 1$ would result in an overall compression ratio of $20: 1$, an acceptably high compression ratio with very little degradation. Further compression of the difference image would result in unacceptable degradation. It is therefore of little value to further reduce the size of the compressed downsampled image. Of course, the further reduction of the minified image could be accomplished by the simple expedient of applying minification yet again along with the DCT compression of the second difference image.

\section{COMPRESSION PARAMETERS}

There are three compression parameters, the energy normalization constant, the percent energy retained, and the frequency weighting coefficient. The energy normalization constant is a number between 5 and 5,000, the percent energy retained is between 1 and 100 , and the frequency weighting coefficient is between 2 and 10. The compression can be controlled using a single number between 1 and 100. The compression control number can be mapped into the three compression parameters to establish the quality of compression. Note that the amount of compression is variable. If there is much detail in the image, the compressed image will be longer than it would be for an image with little detail. The three compression parameters can be developed from the control parameter as 
shown in the following equations:

$$
\begin{aligned}
& \text { Normalization_Constant } \\
& \qquad \begin{array}{l}
=0.5 *(\text { Control_Param })^{2}+5 \\
\text { Percent_Energy }=10 * \text { Sqrt }(\text { Control_Param }) \\
\text { Freq_Weight_Coef } \\
=0.9 * \text { Sqrt }(\text { Control_Param })+1
\end{array}
\end{aligned}
$$

This mapping of the compression control parameter to each of the compression parameters will permit the control of the compression to be exercised very easily on the basis of body part and modality at each site.

\section{COMPRESSION SIGNAL-NOISE RATIO}

The compression noise is the difference between the original image and the reconstructed image. The use of the energy threshold frequency cutoff and the energy normalization level will establish the noise level that is relative to the image energy level. Because the low frequencies are removed in the minified image, the energy that is considered is that of the high frequencies. This removal of the low frequencies before compression makes the compression local; ie, the compression depends not on the image intensity over a large area but on the changes in intensity over a small area. The signal-noise ratio (SNR) can be calculated by considering the noise to be the departure from the difference image.

$$
\begin{aligned}
& \text { SNR_deletion }=1 \\
& \quad-\log (1-\text { proportion_energy_deleted })
\end{aligned}
$$

A second component of the SNR is the quantization noise. If the quantization level is established with reference to the energy level of the block, the quantization noise component can be established by analyses

SNR_Quantization $=10.79$

$$
+10 \log \text { (Normalization_Level), }
$$

where Normalization-Level is the level to which the signal is raised before the quantization is performed. The quantization must, of course, be performed by rounding, not by truncation. Truncation would lose $10.79 \mathrm{~dB}$ of SNR.

The composite SNR is the signal compared with the square root of the sum of the squares of the two noise components. The addition must, of course, be made in the linear domain, not in terms of the SNRs expressed in decibels.

The use of the frequency weighting of the DCT coefficients complicates the calculation of the SNR. The contribution due to the elimination of some of the high-frequency energy stays the same. However, the quantization noise becomes frequency dependent. The SNR for the quantization depends on how the energy of the image is distributed over frequency. From another point of view, the eye averages out the high-frequency noise, thus de-emphasizing this contribution to the noise. The use of the SNR calculated above is appropriate for the perceived noise in the image for all but the highest magnifications.

\section{DISCUSSION}

The use of a very simple lossless compression permits the compression to be performed very quickly, even on software-only computers. The DCT compression with the separate encoding of the difference image provides a high compression ratio with very little degradation of the image quality. The use of the difference image suppresses much of the blocking usually associated with block transform compression. The elimination of the error in the low frequencies along with the frequency weighting of the DCT coefficients pushes the compression error into the high frequencies where it is not easily perceived. The higher level of computation for lossy compression generally will require augmentation of the computer with a high-speed arithmetic processor or an array processor for computation times that are less than several tens of seconds.

The use of the difference image in the compression makes the compression "local." The viewer may manipulate the portion of the $d y$ namic range of the image that is viewed and the size of the area viewed without reference to the general brightness level in the area being viewed. The compression noise does not depend on the general brightness of the area of the image.

The lossless compression is used in the image working storage, providing a larger virtual storage space and a higher data rate to the worksta- 
tions. The lossy compression is used in the image archive. Selected large images will use the lossy compression to reduce the required storage by an order of magnitude.

The lossless compression will be the primary compression for new images moved over a communication network from spokes to hubs. The lossless compression may be executed in any workstation because it can be performed efficiently even in a workstation with no augmented arithmetic.
The lossy compression may be used for moving selected historical images from the spokes to the hubs. It is desirable to have a computer augmented with an array processor so that this compression may be done quickly.

\section{ACKNOWLEDGMENT}

The author thanks Jim Stenstrom for his help in the development and implementation of the approach to radiological image compression.

\section{REFERENCES}

1. Williams RN: Adaptive Data Compression. Boston, MA, Kluwer Academic, 1991

2. Pennebaker W: JPEG Technical Specification, Revision 8. Working Document No. JTC1/SC2/WG10-JPEG8-R8 Consultive Committee on International Telephony and Telegraphy Joint Photography Experts Group, Geneva, Switzerland, August 1990
3. Clarke RJ: Transform Coding of Images. San Diego, CA, Academic, 1985

4. ACR-NEMA: Digital Imaging and Communications. ACR-NEMA Standards Publication No. 300-1985. Washington, DC, National Electrical Manufacturer's Association, 1985

5. Parks TW, Burrus CS: Digital Filter Design. New York, NY, Wiley, 1987, p 73 